\title{
Tumor-targeted liposomal drug delivery mediated by a diseleno bond-stabilized cyclic peptide
}

\author{
This article was published in the following Dove Press journal: \\ International Journal of Nanomedicine \\ II March 2013 \\ Number of times this article has been viewed
}

\section{Chong $\mathrm{Li}^{1,2}$ \\ Yixin Wang ${ }^{3}$ \\ Xiaolin Zhang' \\ Li Deng' \\ Yan Zhang' \\ Zhangbao Chen'}

'Key Laboratory on Luminescence and Real-Time Analysis, Ministry of Education, College of Pharmaceutical Sciences, Southwest University, Chongqing, People's Republic of China; ${ }^{2}$ School of Pharmacy, Key Laboratory of Smart Drug Delivery, Ministry of Education and PLA, Fudan University, Shanghai, People's Republic of China; ${ }^{3}$ Department of Pharmacy, the Second People's Hospital of Sichuan Province and Sichuan Cancer Hospital, Chengdu, People's Republic of China
Correspondence: Chong $\mathrm{Li}$ Key Laboratory on Luminescence and Real-Time Analysis, Ministry of Education, College of Pharmaceutical Sciences,

Southwest University, No 2

Tiansheng Road, Chongqing 4007/6,

People's Republic of China

Tel +86 236825 I 225

Fax +86 236825 I225

Email chongli2009@gmail.com

\begin{abstract}
Peptide ligands have played an important role in tumor-targeted drug delivery as targeting moieties. The in vivo fate of peptide-mediated drug delivery systems and the following antitumor effects may greatly depend on the stability of the peptide ligand. In the current study, a tumor-targeting cyclic peptide screened by phage display, Lyp-1 (a peptide that specifically binds to tumor and endothelial cells of tumor lymphatics in certain tumors), was structurally modified by replacement of the original intramolecular disulfide bond with a diseleno bond. The produced analog Syp-1 (seleno derivative of Lyp-1) maintained specific binding ability to the target protein $\mathrm{p} 32(\mathrm{Kd}=18.54 \mathrm{nM})$, which is similar to that of Lyp- $1(\mathrm{Kd}=10.59 \mathrm{nM})$, indicated by surface plasmon resonance assay. Compared with Lyp-1, Syp-1 showed significantly improved stability against serum. After the peptide attached onto the surface of fluorophore-encapsulating liposomes, the more efficient tumor uptake of liposomal fluorophore mediated by Syp-1 was observed. Furthermore, Syp-1 modified liposomal doxorubicin presented the most potent tumor growth inhibitory ability among all the therapeutic groups, with a low half maximal inhibitory concentration of $588 \mathrm{nM}$ against MDA-MB-435 cells in vitro and a high tumor inhibition rate of $73.5 \%$ in vivo. These findings clearly indicated that Syp-1 was a stable and effective tumor targeting ligand and suggest that the sulfur-to-selenium replacement strategy may help stabilize the phage-displayed cyclic peptide containing disulfide-bond under physiological conditions and strongly support the validity of peptide-mediated drug targeting.
\end{abstract}

Keywords: tumor targeting, liposome, cyclic peptide, selenopeptide

\section{Introduction}

During the past few decades, remarkable progress has been made in the development of a "magic bullet"1 or tumor-targeted drug delivery systems (DDS) due to the application of new biomaterials, identification of new tumor targets, and novel ligands with related mechanisms revealed. ${ }^{2}$ The phenomenon that newly formed tumor vessels harbored abnormal architectures necessitated the sub-micrometer size distribution of DDS for accumulation in tumor tissue (also known as the enhanced permeability and retention effect). Recently, requirements for particle size of DDS have been further investigated for those poorly permeable tumors, ${ }^{3}$ and also expanded at the level of sub-cellular distribution. ${ }^{4}$ In order to lengthen the in vivo circulation time, "stealth" modifications of DDS were performed, among which polyethylene glycol (PEG)-ylation was used most widely and alternatives were under development. ${ }^{5}$ These methods can be summed up as passive tumor-targeting, while active tumor-targeting strategies were developed to enhance the specificity of DDS for tumors. The "bullet" body was prepared using stimuli-sensitive polymer materials to be responsive to a tumor microenvironment such 
as $\mathrm{pH}$ values, ${ }^{6}$ enzymes, ${ }^{7}$ or other factors ${ }^{8}$ for controlled drug release. Furthermore, the use of targeting moiety, which can selectively recognize extracellular biomarkers (mainly proteins), may further improve the targeting abilities of drug carriers for high efficiency and low toxicity in tumor therapy.

There are various kinds of molecules as targeting moieties including small molecules and macromolecules. Among these, peptide ligands have played a crucial role in tumor-targeted drug delivery. ${ }^{9-11}$ The advantages of peptide as a targeting moiety, such as small in size, easy site-specific modification, better organ penetration, low immunogenicity, and target protein binding with high affinity and unsurpassed specificity, have been fully utilized. For decades, the phage display of combinatorial peptide libraries has been used successfully to screen peptides that target specific protein molecules, ${ }^{12}$ cells in vitro, ${ }^{13}$ and even tissues and organs in vivo. ${ }^{14,15}$ The combination of peptide ligand biopanning and further construction of peptide-modified drug carriers can therefore provide an innovative approach to develop active tumor-targeted DDS. ${ }^{16,17}$ Considering the significant influence of biochemical stability of peptides on their bioactivities, ${ }^{18}$ cyclic peptides such as those disulfide-bridged peptides screened by phage display, may generally have a priority over linear peptides with similar chain lengths. However, because disulfide bonds were reversible, to some extent, under physiological conditions, ${ }^{19-21}$ the potential negative effect on the stability of peptides containing disulfide bonds may not be avoided and novel structural modification strategy is worth investigating.

Selenocysteine $(\mathrm{Sec})$, a cysteine analogue with the original sulfur-containing thiol group replaced by a seleniumcontaining selenol group, is called the 21 st natural amino acid. $^{21,22}$ There are over 20 kinds of human selenoproteins, especially enzymes, in which Sec is present as a key building and functional block. Compared with the unique biosynthesis of selenopeptides and selenoproteins, solid-phase chemical synthesis has aroused great interest in the past several decades because of the relatively simple process. Since selenium is a member of the sulfur group on the periodic table of elements, Sec has a structure similar to that of cysteine, and the replacement of disulfide bonds by diseleno bonds may only produce marginal changes in the structure of the peptide and protein. ${ }^{23}$ In addition the higher reduction potential of Sec than cysteine can entail a remarkably enhanced stability of the diseleno linkage against reduction. ${ }^{24}$ Even more attractive is the fact that selenium is an essential micronutrient for human health. ${ }^{25}$ Therefore, the sulfur-toselenium replacement strategy may confer enhanced stability to the phage-displayed cyclic disulfide-bridged peptide under physiological conditions and improve the targeting efficiency of peptide-mediated drug delivery.

The Lyp-1 peptide (a peptide that specifically binds to tumor and endothelial cells of tumor lymphatics in certain tumors), harboring the sequence of CGNKRTRGC via single disulfide connectivity, was originated from a $\mathrm{CX}_{7} \mathrm{C}$ phage display peptide library. ${ }^{26}$ The target of Lyp-1, the p32 protein, was found to be overexpressed not only in a variety of tumor cells but also in tumor-associated lymphatics and macrophages; the function of Lyp-1 as a targeting moiety can therefore be extended to both tumor sites and tumor microenvironments. ${ }^{27-32}$ For example, Lyp-1 modified micelles loaded with artemisinin have shown significant inhibition effects of tumor growth and metastasis via dual-targeting of both MDA-MB-435 melanoma and its lymphatics. ${ }^{32}$ In order to explore the feasibility of a sulfur-to-selenium replacement strategy to improve the biostability of disulfidelinked cyclic peptide while systematically investigating how the stability of disulfide bonds affect the function of such peptide ligands as escort molecules for targeted drug delivery, Lyp-1 peptide was used as the model peptide in the current study. The analog of Lyp-1 with a diseleno bond (Syp-1) was synthesized via solid phase peptide chemistry. Peptide-modified liposomal DDS were prepared accordingly. A systematic comparison between Syp-1 and Lyp-1 as tumor targeting moiety was sequentially performed including the evaluation of serum stability, targeting efficiency, and therapeutic effects both in vitro and in vivo.

\section{Materials and methods Materials}

Fmoc-Sec(Mob)-OH was purchased from Alabiochem (Zhangjiagang, People's Republic of China). Boc- and Fmocamino acids were obtained from GL Biochem (Shanghai, People's Republic of China). Hydrogenated soybean phosphatidylcholine (HSPC) and phosphatidylethanolamine distearoyl methoxy polyethylene glycol conjugate $\left(\mathrm{mPEG}_{2000}{ }^{-}\right.$ DSPE) were purchased from Lipoid GmbH (Ludwigshafen, Germany), and maleimide-derivatized PEG-DSPE (MAL$\mathrm{PEG}_{3400}$-DSPE) was purchased from Laysan Bio (Arab, AL, USA). Sephadex CL-4B, 5-carboxyfluorescein (FAM), and methylthiazolyldiphenyl-tetrazolium bromide (MTT) were purchased from Sigma-Aldrich (St Louis, MO, USA). 1,1'-dioctadecyl-3,3,3,3' tetramethyl indotricarbocyanine iodide (DiR) was purchased from Invitrogen Corp (Life Technologies, Carlsbad, CA, USA). Doxorubicin (DOX) was purchased from Dalian Meilun Biology Tech Co, Ltd (Dalian, People's Republic of China). Recombinant human 
p32 protein was purchased from Sino Biological Inc (Beijing, People's Republic of China). Methanol, acetonitrile, and other high-performance liquid chromatography (HPLC) grade reagents were obtained from Fisher Scientific (Thermo Fisher Scientific, Waltham, MA, USA). Cell culture medium and the supplements were all from Invitrogen Corp. All chemicals were analytic reagent grade.

MDA-MB-435 melanoma cells were kindly provided by Professor Weiyue Lu from Fudan University. The cells were grown in Roswell Park Memorial Institute (RPMI) 1640 medium supplemented with $10 \%(\mathrm{v} / \mathrm{v})$ heat-inactivated fetal bovine serum, $100 \mathrm{U} / \mathrm{mL}$ penicillin, and $100 \mu \mathrm{g} / \mathrm{mL}$ streptomycin. Cultures were maintained under a humidified atmosphere of $5 \% \mathrm{CO}_{2}$ at $37^{\circ} \mathrm{C}$.

Male BALB/c nude mice of age 4-6 weeks were obtained from Shanghai SLAC Laboratory Animal Co, Ltd (Shanghai, People's Republic of China) and kept under specific pathogenfree conditions. All animal experiments were carried out in accordance with the guidelines for animal experiments at Southwest University.

\section{Peptide synthesis}

The Syp-1 peptide was synthesized using the Fmoc method, according to procedures reported in the literature. ${ }^{33}$ Briefly, after chain assembly of Syp-1 (sequence: SecGNKRTRG$\mathrm{Sec}$ ), peptides on resins were treated with trifluoroacetic acid (TFA) and a mixture of TFA- triisopropylsilane-thioanisolewater-dichloromethane (85:5:5:2:3, v/v) at $20^{\circ} \mathrm{C}$. Under these conditions, the peptides were cleaved from the resins, with simultaneous removal of protective groups and formation of diselenide bonds achieved in a single step. The Lyp-1 peptide was synthesized via the Boc solid-phase peptide synthesis strategy as reported in the literature. ${ }^{29}$

\section{Peptide purification and characterization}

All crude peptides were purified to homogeneity by reversedphase HPLC before further usage. Due to the sticky property of Syp-1, HPLC analysis was performed at $40^{\circ} \mathrm{C}$ on a $\mathrm{C} 4 \mathrm{col}-$ umn using a linear gradient of 5\%-65\% acetonitrile containing $0.1 \%$ TFA at a flow rate of $1 \mathrm{~mL} /$ minute for 30 minutes and HPLC purification was performed using a linear gradient of $20 \%-45 \%$ acetonitrile containing $0.1 \%$ TFA at a flow rate of $10 \mathrm{~mL} /$ minute for 60 minutes. The purified peptides were characterized by electrospray ionization mass spectrometry (ESI-MS). ESI-MS was performed on an ESI-TRAP Esquire 3000 plus mass spectrometer (Bruker, Billerica, MA, USA). Analyses were performed in the positive mode using watermethanol $(50 / 50, \mathrm{v} / \mathrm{v})$ as eluent.

\section{Surface plasmon resonance detection}

The experiment was conducted on the platform of PlexArray ${ }^{\circledR}$ HT (Plexera LLC, Seattle, WA, USA). The surface initiative polymerization surface was activated by mixture of $0.4 \mathrm{M}$ 1-Ethyl-3-(3-dimethylaminopropyl)carbodiimide and $0.1 \mathrm{M}$ $\mathrm{N}$-hydroxysuccinimide for 15 minutes at room temperature. One microgram per milliliter of Lyp-1 and Syp-1 were immediately pipette-spotted on the activated surface, and incubated at $4^{\circ} \mathrm{C}$ for 60 minutes. Thereafter, the chip was incubated with $1 \mathrm{M}$ ethanolamine- $\mathrm{HCl}(\mathrm{pH} 8.5)$ for 10 minutes to block the remaining activated $\mathrm{N}$-hydroxysuccinimide groups. HSB-EP buffer (10 mM HEPES, $150 \mathrm{mM} \mathrm{NaCl}, 3 \mathrm{mM} \mathrm{NaCl}, 3 \mathrm{mM}$ ethylenediaminetetraacetic acid, and $0.01 \% \mathrm{P} 20, \mathrm{pH} 7.4$ ) was used as a running buffer. Series dilutions of various concentrations of $\mathrm{p} 32$ protein were prepared and injected over the chip surface. Analytes were injected into flow cells at the rate of $1 \mathrm{uL} /$ second for 200 seconds of association, followed with 300 seconds of dissociation. Subsequently, the surface was regenerated with 1:200 (v/v) $\mathrm{H}_{3} \mathrm{PO}_{4}$ at $5 \mathrm{uL} /$ second for 150 seconds to wash off all bound analytes. Then the surface was ready for the next cycle of injection. The whole experiments were conducted at $25^{\circ} \mathrm{C}$.

\section{Serum stability assay}

The assay was initiated by adding the test peptide (Syp-1 or Lyp-1) to cell culture medium RPMI 1640 containing 25\% fetal bovine serum at $37^{\circ} \mathrm{C}$ with a final peptide concentration of $100 \mu \mathrm{M}$. Two-hundred-fifty microliter samples of the respective peptides were removed from the incubations at predetermined intervals for 12 hours. For each sample, the obtained aliquot was treated by $5 \%$ TFA to precipitate serum proteins and the supernatant after centrifugation was collected and analyzed using HPLC. This assay was performed in triplicate.

\section{Preparation and characterization of liposomes} Synthesis of SyP-I-PEG ${ }_{3400}$-DSPE and LyP-I-PEG ${ }_{3400}$-DSPE

Lyp-1-PEG ${ }_{3400}$-DSPE was synthesized as reported in the literature. ${ }^{29}$ Briefly, the linear S-acetomidomethyl (Acm)-protected LyP-1 $\left(\mathrm{CC}_{(\mathrm{Acm})}\right.$ GNKRTRG $\mathrm{C}_{(\mathrm{Acm})}$, LyP$\left.1_{(2 \mathrm{Acm})}-\mathrm{Cys}\right)$ was covalently linked to a maleimide group of MAL-PEG-DSPE via the thiol group of the N-terminal cysteine. The molar ratio of peptide versus MAL-PEGDSPE was 1.2:1. After dialysis against distilled water to remove free peptide, the resulting LyP-1 $1_{(2 \mathrm{Acm})}$-PEG-DSPE was further treated by iodine oxidation for deprotection of 
the Acm-protected thiol group and simultaneous formation of a disulfide bond. The reaction solution was dialyzed again to remove salts and then lyophilized.

For coupling Syp-1 with MAL-PEG ${ }_{3400}$-DSPE, thiolated Syp-1 (sequence: CSecGNKRTRGSec), which has a free thiol group of cysteine at the N-terminal was synthesized using the Fmoc method similar to that mentioned above. Then Syp-1-PEG ${ }_{3400}$-DSPE was synthesized through the reaction of the thiol group with the maleimide group. ${ }^{34}$

The progress of the reaction was monitored by determining the noncoupled peptide fraction using HPLC. The successful conjugation of peptide with MAL-PEG ${ }_{3400}$-DSPE was confirmed via ${ }^{1} \mathrm{H}-\mathrm{NMR} .{ }^{29}$

\section{Preparation of liposomes}

Syp-1-PEG-liposomes loading FAM, DiR, or DOX (S-P-LS/ FAM, S-P-LS/DiR, or S-P-LS/DOX) were prepared by the rotation evaporation and extrusion method for different uses. $^{28,29}$ For FAM-loaded liposomes, HSPC, cholesterol, $\mathrm{mPEG}_{2000}$-DSPE, and Syp-1-PEG ${ }_{3400}$-DSPE were dissolved in chloroform/methanol $(3: 1 \mathrm{v} / \mathrm{v})$ at a molar ratio of 55:45:2:1. After the organic solvents were volatilized entirely to form a thin film at $37^{\circ} \mathrm{C}$, FAM solution was added for hydration at $60^{\circ} \mathrm{C}$ for 2 hours. The obtained suspension was repeatedly extruded through polycarbonate membrane filters with a pore diameter of $100 \mathrm{~nm}, 80 \mathrm{~nm}$, and $50 \mathrm{~nm}$ successively, using a mini-extrusion device (Avanti Polar Lipids, Alabaster, AL, USA). Then liposomes were purified through a Sephadex CL-4B size exclusion column equilibrated with saline buffer $(\mathrm{pH}$ 7.4) to remove free FAM and the eluent was collected. The procedure for preparation of DiR-loaded liposomes was similar to that of FAM-loaded liposomes, except that DiR was dissolved in chloroform/methanol $(3: 1 \mathrm{v} / \mathrm{v})$ with those lipid membrane materials. S-P-LS/DOX was prepared by the typical ammonium sulfate gradient method.

Lyp-1-PEG-liposomes loading FAM, DiR, or DOX (L-P-LS/FAM, L-P-LS/DiR, or L-P-LS/DOX) and PEGLiposomes loading FAM, DiR, or DOX (P-LS/FAM, P-LS/ DiR, or P-LS/DOX) were prepared in parallel.

\section{Characterization of liposomes}

The particle size, the polydispersity index, and the zeta potential for each of these liposomes were determined using the dynamic light scattering method (Nano ZS; Malvern Instruments, Malvern, UK). For FAM-loaded or DiR-loaded liposomes, the concentrations of the encapsulated fluorescence probes were measured by a fluorescence spectrophotometer (F-4500, Hitachi, Tokyo, Japan)
(FAM at Ex/Em 494/522 $\mathrm{nm}$ and DiR at 741/776 nm), after treatment with $10 \%$ Triton-X100 to release the vesicular structures' contents. For DOX-loaded liposomes, concentrations of encapsulated DOX were detected by the reversed phase HPLC method after treatment with Triton-X100.

To determine the encapsulation efficiency of DOX in S-P-LS/DOX, the obtained liposomes were divided into two equal parts before chromatographic purification. One part was purified on a Sephadex CL-4B column. The eluent was collected and the encapsulated DOX was measured by HPLC. The other half was diluted into the same volume of the eluent above. Then, the total amount of DOX was detected. The encapsulation efficiency of the liposomes was calculated as the encapsulated DOX divided by the total amount of DOX. Other liposomes were determined in parallel.

\section{Targeting ability study of liposomes}

\section{Cellular uptake of liposomes by tumor cells}

The MDA-MB-435 melanoma cells were plated at $1 \times 10^{5}$ cells/well in 24 -well plates and incubated overnight at $37^{\circ} \mathrm{C}$, maintained in RPMI 1640 medium containing 10\% (v/v) heat-inactivated fetal bovine serum, $100 \mathrm{U} / \mathrm{mL}$ penicillin, and $100 \mu \mathrm{g} / \mathrm{mL}$ streptomycin under a humidified atmosphere of $5 \% \mathrm{CO}_{2}$. Then the cells were incubated with S-P-LS/FAM, L-P-LS/FAM, P-LS/FAM, or FAM in culture medium for 2 hours at the concentration of $1 \mu \mathrm{M}$ (FAM). Cells were then washed with phosphate-buffered saline (PBS) ( $\mathrm{pH} 7.4)$ three times to remove free liposomes or FAM, and visualized using a fluorescent microscope (Olympus Corporation, Tokyo, Japan). For quantitative analysis, cells were maintained and treated using the same steps as described above. After incubation with liposomes for 2 hours, cells were harvested by centrifugation, washed three times with ice-cold PBS and resuspended in PBS. The fluorescence intensity was detected by counting $1 \times 10^{4}$ cells per sample each time via fluorescence activated cell sorting (BD FACS Aria; BD Biosciences, San Jose, CA, USA). This flow cytometry assay was performed in triplicate.

To further investigate the potential influence of peptide stability on the targeting ability of peptide-modified liposomes, a preliminary analysis was conducted. The liposome samples were pre-exposed to serum for 1 hour prior to their addition to the cells. The final lipid concentration of the samples was $0.25 \mathrm{mg} / \mathrm{mL}$. Then pre-treated samples were added to the cells in serum-free medium for 2 hours at $37^{\circ} \mathrm{C}$. The cellular uptake of liposomes by tumor cells was evaluated by the methods mentioned above. 


\section{Liposomes uptake by tumor in vivo}

The MDA-MB-435 tumor model was established by subcutaneous inoculation with $1 \times 10^{7}$ cells via the right shoulder blade in nude mice. Tumor volumes were calculated on the basis of caliper measurements using the following formula: Tumor volume $=\left[\right.$ length $\left.\times(\text { width })^{2}\right] / 2$. To evaluate tumor-targeting efficiency, the mice were mock-treated with fluorophoreencapsulating liposomes $(100 \mu \mathrm{L}$ liposome with $20 \mu \mathrm{g} / \mathrm{mL}$ DiR) via the tail vein after the tumor had been established for 4 weeks with sizes of $\sim 150 \mathrm{~mm}^{3}$ on average. At a predetermined time point, the mice were anesthetized and the distribution of liposomal DiR was detected using an in vivo imaging system (FX Pro; Kodak, Rochester, NY, USA) equipped with a $730 \mathrm{~nm}$ excitation filter and a $790 \mathrm{~nm}$ emission band pass filter set. For ex vivo imaging of separated organs, mice were sacrificed by cervical dislocation. Three mice were used in parallel each time.

\section{Pharmacodynamic evaluation}

\section{MTT cell viability assay}

The antitumor activities of DOX-loaded liposomes against MDA-MB-435 cells were evaluated by MTT colorimetric assay performed in triplicate. Three-thousand cells per well were seeded in 96-well plates at $200 \mu \mathrm{L}$ per well and allowed to grow for 24 hours before being treated with various liposomes. Then, cells were incubated with culture medium containing liposomes with a series of DOX concentrations for 72 hours. Percent cell viability was determined on the basis of optical density values of sample wells versus reference wells (Tecan Group Ltd, Männedorf, Switzerland). This assay was performed in triplicate.

\section{Tumor growth inhibition study}

The tumor-bearing nude mice model was established as demonstrated above. Treatment of mice was initiated (ie, day 0 ) after the tumor had been established for 4 weeks. A total of 24 mice were randomly assigned to four groups $(n=6)$ and treated with blank PEG-liposome, P-LS/DOX, L-P-LS/DOX, and S-P-LS/ DOX, respectively. Different formulations $(200 \mu \mathrm{L})$ were administered on the 2nd, 5th, 8th, and 11th day, with a dose of $3 \mathrm{mg} / \mathrm{kg}$ every 3 days by intravenous injections via the tail vein. The volumes of the tumors were measured every other day. The tumor inhibition rate was calculated using the formula inhibition rate $(\%)=(1-\mathrm{Wt} / \mathrm{Wc}) \times 100$, where $\mathrm{Wt}$ and $\mathrm{Wc}$ are the mean tumor weight of the treated and control groups, respectively.

\section{Data analysis}

Nonlinear regression analysis was performed using GraphPad Prism 5.0 (GraphPad Software, San Diego, CA, USA) to calculate the Kd values and half maximal inhibitory concentration values presented as mean \pm standard deviation. Statistical data analysis was performed using the Student's $t$-test and $P<0.05$ was considered statistically significant.

\section{Results Preparation and characterization of Syp-I peptide}

The purity and molecular weight of the synthesized peptides were confirmed by HPLC and ESI-MS respectively (see Figure S1 for Syp-1 peptide). The binding affinities of peptides for $\mathrm{p} 32$ protein were detected by the surface plasmon resonance method. Peptides were immobilized on the surface initiative polymerization surface chip with triplicate spots. Series dilutions of various concentrations of $\mathrm{p} 32$ protein ( $50 \mathrm{nM}, 100 \mathrm{nM}, 200 \mathrm{nM}$, and $400 \mathrm{nM}$ ) were injected into the flow cells. The binding signals are shown in Figure 1. The binding curves were double-referenced with both blank surface spot and running buffer as a blank analyte. Then binding affinities of p32-Syp-1 and p32-Lyp-1 were calculated as $18.54 \mathrm{nM}$ and $10.59 \mathrm{nM}$, respectively, after being fitted with the 1:1 Langmuir binding model (See Table S1).

\section{Preparation and characterization of Syp-I modified material}

The peptide modified membrane material was synthesized as a key ingredient for tumor-targeting liposome preparation. The coupling strategy used here was the reaction between the maleimide and thiol groups because it is rapid and proceeds close to completion under mild reaction conditions. ${ }^{35}$ As shown in Figure 2, the characteristic peak of the maleimide group of MAL-PEG ${ }_{3400}-\mathrm{DSPE}$ was at $6.7 \mathrm{ppm}$. This maleimide peak disappeared in the ${ }^{1} \mathrm{H}-\mathrm{NMR}$ spectrum of Syp-1-PEG ${ }_{3400}{ }^{-}$ DSPE, indicating the successful conjugation of Syp-1 with MAL-PEG $_{3400}$-DSPE. For synthesis of Lyp-1-PEG $3400-\mathrm{DSPE}$, a much more complicated procedure was performed with protection and deprotection of Acm groups and iodine oxidation successively, in case shuffling of the disulfide bonds occurred in thiolated Lyp-1 before conjugation to MAL-PEG-DSPE. For the three kinds of materials (MAL-PEG ${ }_{3400}$-DSPE, Syp1-PEG ${ }_{3400}$-DSPE, and Lyp-1-PEG 3400 -DSPE), the methylene protons of DSPE had multiple peaks around $1.26 \mathrm{ppm}$ and repeating units of PEG appeared in the range of 3.7-3.8 ppm. ${ }^{29}$ The solvent peak of $\mathrm{CDCl}_{3}$ was observed at $7.26 \mathrm{ppm}$.

\section{Characterization of S-P-LS}

The average size of S-P-LS/DOX was $87.14 \mathrm{~nm}$, and the polydispersity index was 0.095 . The entrapment efficiency of 
A
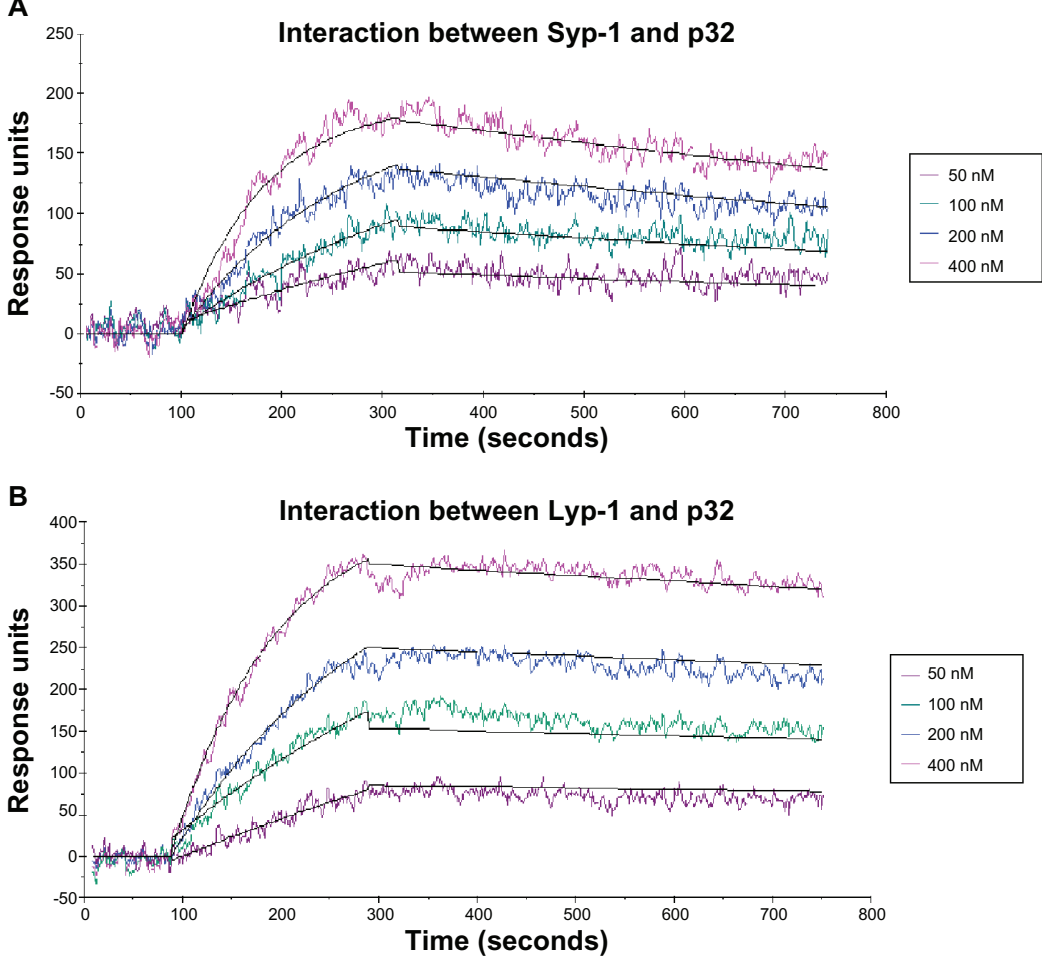

Figure I The binding curves between peptide ligands and p32 protein and the I:I Langmuir model fitting (response units increase as the concentration of $p 32$ protein increases from $50 \mathrm{nM}$ to $400 \mathrm{nM}$ ). (A) Interaction between Syp-I and p32; (B) Interaction between Lyp-I and p32.

S-P-LS/DOX was 96.3\%. Other liposomes were determined in parallel (see Table S2).

\section{Evaluation of Syp-I as a targeting moiety for melanoma \\ Serum stability of Syp-I versus Lyp-I}

According to HPLC analysis as shown in Figure 3, Lyp-1 was quantitatively degraded within 12 hours, whereas Syp-1 showed a relatively slow decreasing trend. During the first hour, more

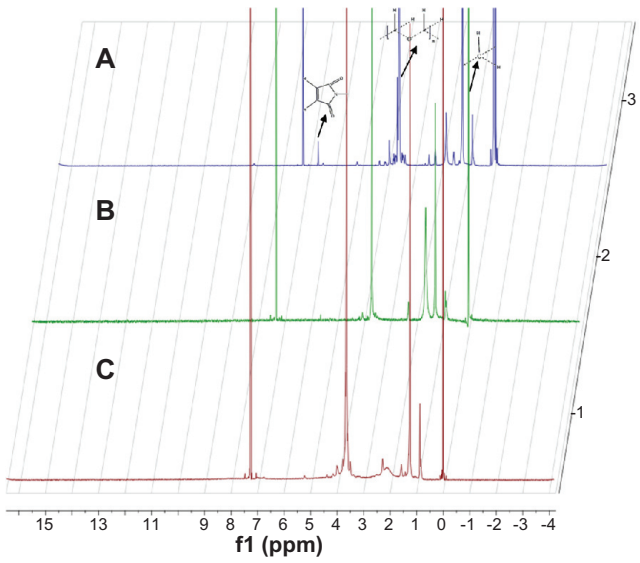

Figure 2 'H-NMR spectra of (A) MAL-PEG ${ }_{3400}-\mathrm{DSPE}$, (B) Lyp-I-PEG ${ }_{3400}-\mathrm{DSPE}$, and (C) Syp-I-PEG 3400 -DSPE at $400 \mathrm{MHz}$.

Abbreviations: MAL-PEG 3400 -DSPE, maleimide-derivatized PEG-DSPE; PEG-DSPE, phosphatidylethanolamine distearoyl methoxypolyethylene glycol conjugate. than $60 \%$ loss of Lyp-1 was observed. By contrast, less than $20 \%$ degradation of Syp-1 was detected. This preliminary results suggested potential superiority of Syp-1 over Lyp-1 with respect to proteolytic stability in vivo.

\section{Tumor cell uptake of S-P-LS in vitro}

The specificity of S-P-LS to MDA-MB-435 cells was evaluated qualitatively by fluorescent microscopy studies and quantitatively by fluorescence intensity detection of FAM

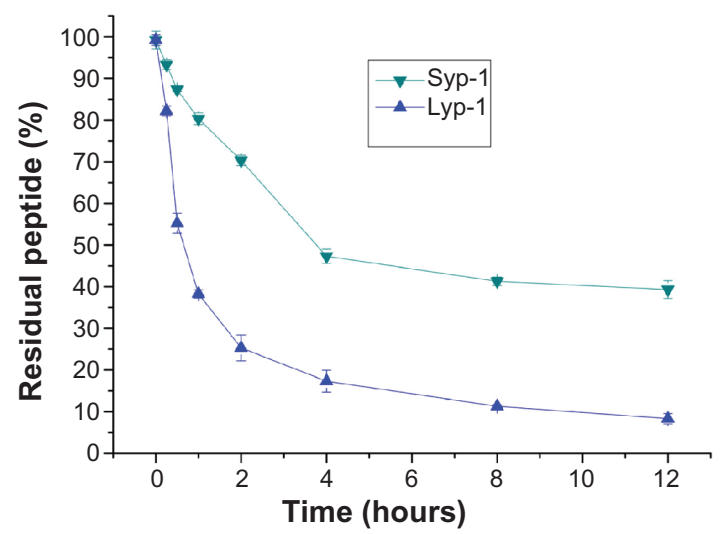

Figure 3 Proteolytic stability of Lyp-I and Syp-I against serum.

Notes: Peptide stability was calculated by dividing the residual amount by the original amount at predetermined time intervals (expressed as percent residual peptide). This assay was detected by HPLC and performed in triplicate.

Abbreviation: HPLC, high performance liquid chromatography. 
positive cells (Figure 4). Both S-P-LS/FAM and L-P-LS/ FAM were efficiently internalized by MDA-MB- 435 cells compared with P-LS/FAM. As shown in Figure 4C, the mean fluorescence intensity of positive cells of S-P-LS/FAM was 1052, significantly higher than that of L-P-LS/FAM (1052 versus $827, P<0.05$ ). Peptide-modified liposomes were also tested for the effect of serum components on their interaction with cells. Fluorescence images showed that internalization of L-P-LS/FAM by MDA-MB-435 cells (Figure 4B) was greatly diminished following 1 hour pre-incubation of formulations with serum. And a 48\% decrease in fluorescence signal was detected quantitatively by fluorescence activated cell sorting, indicating less interaction of Lyp-1-modified liposomes with cells. This may be attributed to the fact that Lyp-1 on the surface of the liposome was cleaved by serum proteolytic enzymes and liposome binding to cells was prevented. For S-P-LS/FAM, even after pre-incubation treatment with serum, efficient liposome internalization was observed with more than $90 \%$ fluorescence signal maintained.

\section{In vivo targeting ability of S-P-LS}

The in vivo targeting efficiency of S-P-LS to melanoma was studied by delivery of liposomal DiR to nude mice bearing MDA-MB-435 tumor via the tail vein. Attachment of the peptide to the liposome surface results in remarkable improvements in tumor targeting of fluorophoreencapsulating liposomes. The accumulation of S-P-LS/DiR in the tumor site was significantly more than that of L-P-LS/ $\mathrm{DiR}$ at 24 hours post-injection (shown in Figure 5A). The same trend was observed for the comparison of isolated tumors among different groups when mice were sacrificed (shown in Figure 5B).

\section{Evaluation of Syp-I-mediated drug delivery and antitumor effect}

In vitro anti-tumor activity of S-P-LS/DOX was evaluated in MDA-MB-435 cells by MTT assay. It can be seen in Figure 6 that a potent tumor cell growth inhibition was found in the S-P-LS/DOX with a low half maximal inhibitory
A

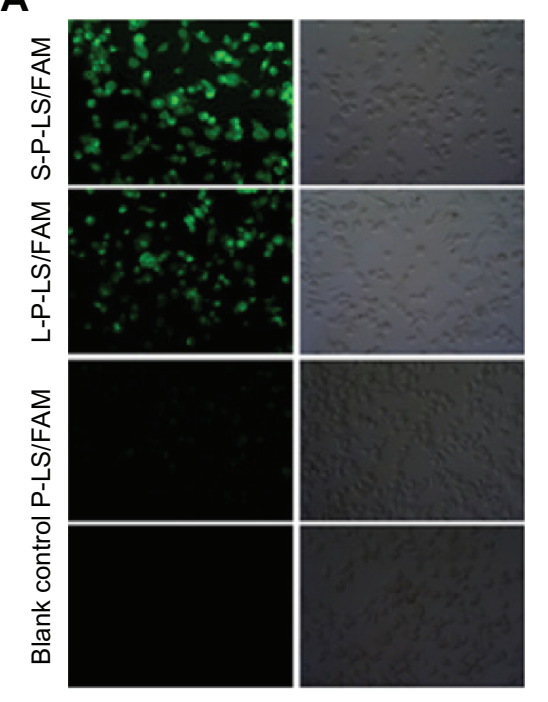

C

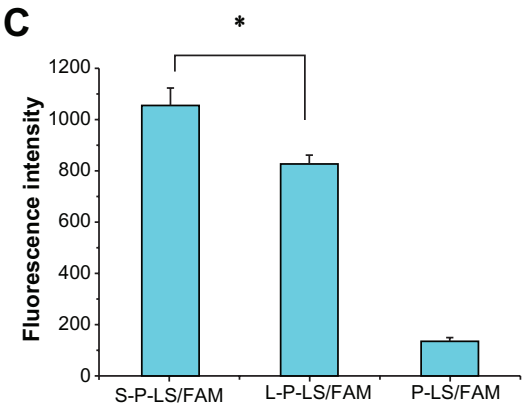

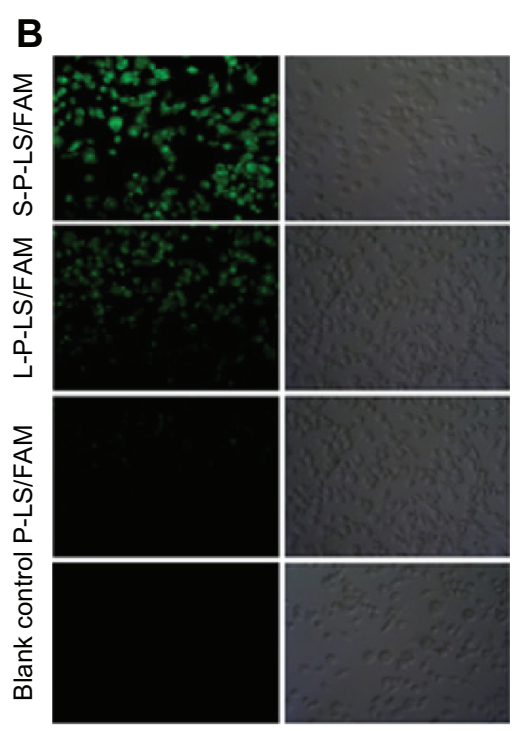

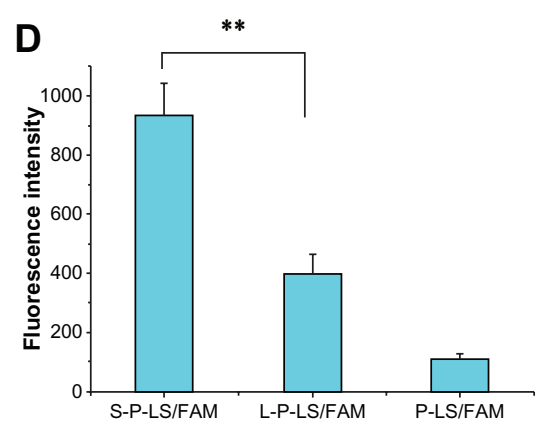

Figure 4 (A and $\mathbf{C})$ : Quantitative and qualitative analysis of the internalization of different FAM-containing liposomes by MDA-MB-435 cells in culture medium for 2 hours at the concentration of I $\mu$ M (FAM). (B and D): Analysis after pre-incubation with serum for I hour at $37^{\circ} \mathrm{C}$.

Notes: These quantitative assays were performed in triplicate. Data represented as mean $\pm S D(n=3)$. $* P<0.05 ; * * P<0.01$.

Abbreviations: FAM, 5-carboxyfluorescein; SD, standard deviation; P-LS/FAM, PEG-liposomes loading FAM; S-P-LS/FAM, Syp-I-PEG-liposomes loading FAM; L-P-LS/FAM, Lyp-I-PEG-liposomes loading FAM; PEG, polyethylene glycol. 


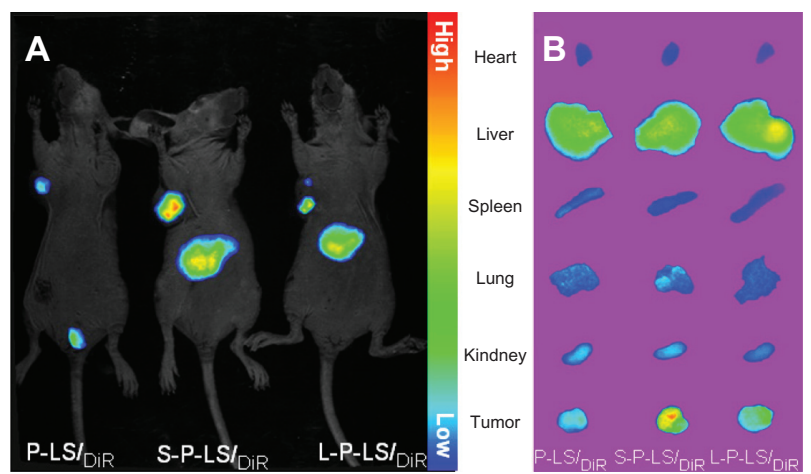

Figure 5 In vivo near-infrared fluorescence imaging of xenografts in nude mice after intravenous injection of DiR-loaded liposomes.

Abbreviations: DiR, I, I'-dioctadecyl-3,3,3,3' tetramethyl indotricarbocyanine iodide; P-LS/DiR, PEG-liposomes loading DiR; S-P-LS/DiR, Syp-I-PEG-liposomes loading DiR; L-P-LS/DiR, Lyp-I-PEG-liposomes loading DiR; PEG, polyethylene glycol.

concentration value of $588 \mathrm{nM}$ compared to $1.48 \mu \mathrm{M}$ of L-P-LS/DOX and 2.67 $\mu \mathrm{M}$ of S-P-LS/DOX. The liposomal DOX with surface modification of Syp-1 showed significantly enhanced cytotoxicity against tumor cells $(P<0.05$ versus L-P-LS/DOX and $P<0.01$ versus $\mathrm{P}-\mathrm{LS} / \mathrm{DOX})$.

Furthermore, the in vivo antitumor efficacy of S-P-LS/ DOX and L-P-LS/DOX were compared in MDA-MB435 melanoma-bearing nude mice. As shown in Figure 7, the tumor size in animals of the blank liposome group increased by near 12 -fold over a period of 2 weeks while S-P-LS/DOX showed the most potent tumor growth inhibition rate of $73.5 \%$ among all the therapeutic groups $(P<0.05$ versus L-P-LS/ DOX of $58.8 \%$ and $P<0.01$ versus P-LS/DOX of $45.1 \%$ ).

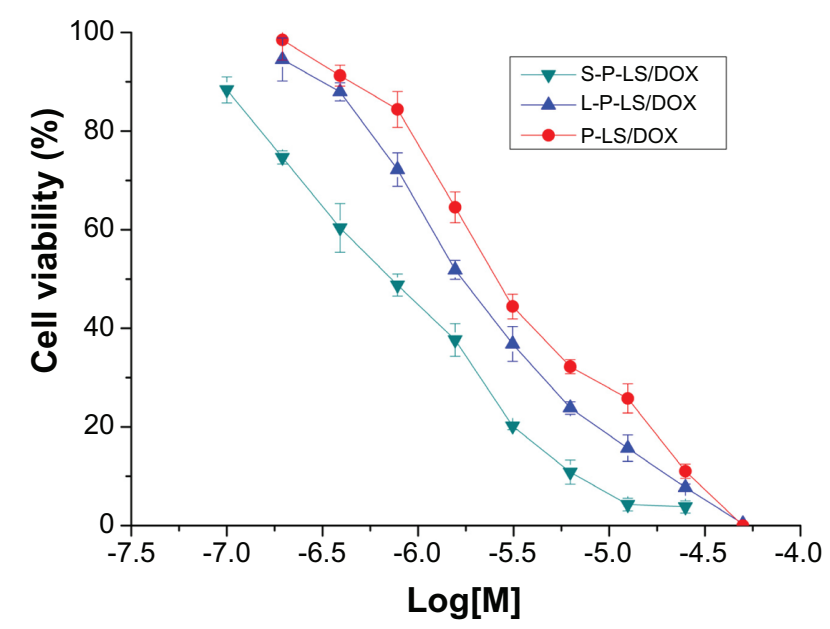

Figure 6 In vitro antitumor activity of liposomal doxorubicin against MDA-MB435 cells was evaluated by MTT colorimetric assay and performed in triplicate. Notes: Relative cell viability was determined on the basis of optical density values of sample wells versus reference wells. Data represented as mean \pm SD $(n=3)$.

Abbreviations: MTT, methylthiazolyldiphenyl-tetrazolium bromide; SD, standard deviation; DOX, doxorubicin; S-P-LS/DOX, Syp-I-PEG-liposomes loading DOX; P-LS/DOX, PEG-liposomes loading DOX; L-P-LS/DOX, Lyp-I-PEG-liposomes loading DOX; PEG, polyethylene glycol.

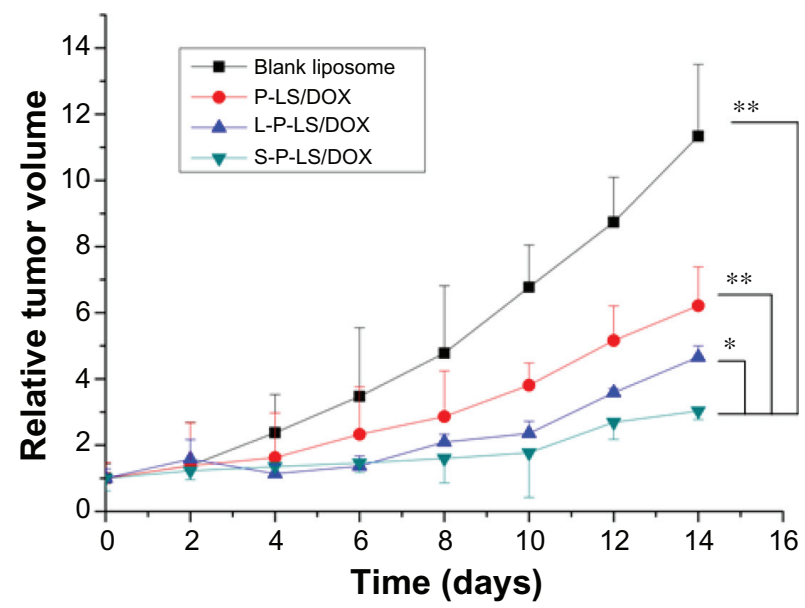

Figure 7 Antitumor efficacy of liposomal DOX in vivo.

Notes: $P<0.01$ for S-P-LS/DOX versus $P$-LS/DOX and $P<0.05$ for S-P-LS/DOX versus L-P-LS/DOX. Data represented as mean $\pm S D(n=6)$. $* P<0.05 ; * * P<0.01$. Abbreviations: DOX, doxorubicin; S-P-LS/DOX, SyP-I-PEG-liposomes loading DOX; P-LS/DOX, PEG-liposomes loading DOX; L-P-LS/DOX, Lyp-I-PEG-liposomes loading DOX; SD, standard deviation; PEG, polyethylene glycol.

\section{Discussion and conclusion}

Lyp-1 was one of the representative disulfide-linked cyclic peptides screened by phage display, which was used as a tumor-targeting moiety recently. ${ }^{28,29}$ In the current study, a structural modification by replacement of the original disulfide bond with a diseleno bond was explored in order to improve the in vivo stability of Lyp-1 while maintaining its function. According to the results of the surface plasmon resonance detection and serum stability assay, Syp-1, the produced analog of Lyp-1, varied only less than two-fold in binding to the target protein $\mathrm{p} 32$ and significantly improved stability against serum compared with Lyp-1, thus presenting a structural base necessary to mediate efficient drug targeting. Thereafter, in order to prepare Syp-1-modified liposomal DDS, a typical modification method was performed where the peptide ligand was thiolated firstly by addition of a terminal cysteine via solid phase chemistry and then conjugated to PEG-DSPE through the reaction of the thiol group with a maleimide group. For Lyp-1, however, a direct addition of cysteine to the peptide sequence may not be acceptable due to possible disulfide rearrangement (known as disulfide shuffling), which necessitated a much more complicated procedure as mentioned above. Besides, it may also be difficult to construct Lyp-1-modified liposomes via the post-modification method because of the harsh reaction conditions for Acm groups' deprotection and iodine oxidation. Therefore, the relative simplicity of the liposome surface modification process indicated the superiority of Syp-1 over Lyp-1 in another aspect. Finally, a systematic comparison of Syp-1 versus Lyp-1 to mediate tumor-targeted 
delivery of liposomal fluorophore or DOX was conducted. Syp-1 efficiently mediated tumor uptake of liposomal DOX and showed significantly enhanced antitumor effects, which is a direct reflection of the improved comprehensive property of Syp-1 over Lyp-1.

In recent decades, great progress has been made in the chemistry of selenopeptide and selenoprotein, which provide a novel route for efficient preparation (total synthesis and folding), functional research, structural analysis, and even potential clinical application of peptides and proteins..$^{23,36-41}$ For example, the potential of preformed peptidyl $\alpha$-selenoesters in native chemical ligation has been explored to mediate rapid reaction at intractable sites. ${ }^{36}$ Meanwhile, researchers have investigated several toxins such as apamin and conotoxin for modification via partial or full replacement of disulfide bonds by diseleno bonds. ${ }^{23,39-41}$ The produced analogs were more stable than their native counterparts and structural analysis showed that the slightly larger atomic radius of selenium had no significant impact on the overall structure. ${ }^{23}$ It was reported that disulfide bonds occurred in nearly $30 \%$ of proteins,${ }^{42}$ and many such peptides and proteins had important physiological functions. As a tumor-targeting moiety, a variety of disulfide-constrained peptides exist, especially those screened by phage display, such as CSNRDARRC peptide for bladder cancer, ${ }^{43}$ CMGNKRSAKRPC for rhabadominosarcoma, ${ }^{44}$ and CASPSGALRSC for breast cancer. ${ }^{45}$ However, the relatively harsh physiological environment in vivo may constitute a potential hurdle to disulfide-constrained peptide-mediated drug targeting. For example, disulfide linkage was reported to be rapidly cleaved in the brain, which may restrict the application of such ligands for recognizing glioma or metastatic brain tumors. ${ }^{46}$ Therefore, further stabilization of the cyclic structure may be considered in order to improve the biostability of disulfide-linked peptide ligands and the following antitumor effects of peptide-mediated drug targeting.

It has been clearly demonstrated that the modification of DDS using tumor-targeting molecules results in increased efficacy, however, it was not fully elucidated whether the mere presence of a ligand influences the in vivo behavior of DDS because in most studies a comparison has been made between DDS with ligands and without ligands. ${ }^{47}$ In the present study, the primary evaluations of two peptide ligands which were aimed at the same target were performed in parallel under limited experimental conditions. Compared with its native counterpart, Syp-1 worked as a more stable and efficient ligand to mediate tumor-targeted drug delivery, suggesting that the biostability of peptide ligands may alter the targeting abilities of drug-loaded liposomes with a PEG density on the particle of $3 \mathrm{~mol} \%$ under certain circumstances. In conclusion, our results have demonstrated a possibility that the sulfur-toselenium replacement strategy can be used to help construct more efficient DDS targeting neoplasms, such as a combination with disulfide-constrained peptide biopanning, and may provide useful information to further understand how the DDS was mediated by targeting ligands to treat tumors.

\section{Acknowledgments}

The authors are grateful for financial support from the National Natural Science Foundation of China (number 81102404 and number 21272187), the National Creating New Drug Program of China (number 2010 ZX 09401-3061-4), the Doctoral Fund of Ministry of Education of China (20110182120015), the Natural Science Foundation of Chongqing (cstc2011jjA1585), a special fund of the Chongqing Key Laboratory (CSTC), Foundation for University Key Teachers by the Chongqing Municipal Education Commission (2011), the Fundamental Research Funds for the Central Universities (XDJK2013A010), the doctoral fund of the Southwest University (SWU110028) and School of Pharmacy, Fudan University, and The Open Project Program of Key Lab of Smart Drug Delivery (Fudan University), Ministry of Education, People's Republic of China (2011SDD-05).

\section{Disclosure}

The authors report no conflicts of interest in this work.

\section{References}

1. Strebhardt K, Ullrich A. Paul Ehrlich's magic bullet concept: 100 years of progress. Nat Rev Cancer. 2008;8(6):473-480.

2. Haley B, Frenkel E. Nanoparticles for drug delivery in cancer treatment. Urol Oncol. 2008;26(1):57-64.

3. Cabral H, Matsumoto Y, Mizuno K, et al. Accumulation of sub-100 nm polymeric micelles in poorly permeable tumours depends on size. Nat Nanotechnol. 2011;6(12):815-823.

4. Pan L, He Q, Liu J, et al. Nuclear-targeted drug delivery of TAT peptideconjugated monodisperse mesoporous silica nanoparticles. J Am Chem Soc. 2012;134(13):5722-5725.

5. Barbé C, Bartlett J, Kong L, et al. Silica particles: a novel drug-delivery system. Adv Mater. 2004;16(21):1959-1966.

6. Chang G, Li C, Lu W, Ding J. N-Boc-histidine-capped PLGA-PEG-PLGA as a smart polymer for drug delivery sensitive to tumor extracellular $\mathrm{pH}$. Macromol Biosci. 2010;10(10):1248-1256.

7. Chau Y, Tan FE, Langer R. Synthesis and characterization of dextranpeptide-methotrexate conjugates for tumor targeting via mediation by matrix metalloproteinase II and matrix metalloproteinase IX. Bioconjug Chem. 2004;15(4):931-941.

8. Cho H, Bae J, Garripelli VK, Anderson JM, Jun HW, Jo S. Redox-sensitive polymeric nanoparticles for drug delivery. Chem Commun (Camb). 2012;48:6043-6045.

9. Dharap SS, Wang Y, Chandna P, et al. Tumor-specific targeting of an anticancer drug delivery system by LHRH peptide. Proc Natl Acad Sci US A. 2005;102(36):12962-12967. 
10. Hatakeyama S, Sugihara K, Shibata TK, et al. Targeted drug delivery to tumor vasculature by a carbohydrate mimetic peptide. Proc Natl Acad Sci U S A. 2011;108(49):19587-19592.

11. Cai LL, Liu P, Li X, et al. RGD peptide-mediated chitosan-based polymeric micelles targeting delivery for integrin-overexpressing tumor cells. Int J Nanomedicine. 2011;6:3499-3508.

12. Lupold SE, Rodriguez R. Disulfide-constrained peptides that bind to the extracellular portion of the prostate-specific membrane antigen. Mol Cancer Ther. 2004;3(5):597-603.

13. Rasmussen UB, Schreiber V, Schultz H, Mischler F, Schughart K. Tumor cell-targeting by phage-displayed peptides. Cancer Gene Ther. 2002;9(7):606-612.

14. Pasqualini R, Ruoslahti E. Organ targeting in vivo using phage display peptide libraries. Nature. 1996;380(6572):364-346.

15. Chen Y, Shen Y, Guo X, et al. Transdermal protein delivery by a coadministered peptide identified via phage display. Nat Biotechnol. 2006;24(4):455-460.

16. Krumpe LR, Mori T. The use of phage-displayed peptide libraries to develop tumor-targeting drugs. Int J Pept Res Ther. 2006;12(1): 79-91.

17. Ruoslahti E, Bhatia SN, Sailor MJ. Targeting of drugs and nanoparticles to tumors. Eur J Cell Biol. 2010;188(6):759-768.

18. Koren E, Apte A, Sawant RR, Grunwald J, Torchilin VP. Cellpenetrating TAT peptide in drug delivery systems: proteolytic stability requirements. Drug Deliv. 2011;18(5):378-384.

19. Schiavo G, Papini E, Genna G, Montecucco C. An intact interchain disulfide bond is required for the neurotoxicity of tetanus toxin. Infect Immun. 1990;58(12):4136-4141.

20. Chen X, Bai Y, Zaro JL, Shen WC. Design of an in vivo cleavable disulfide linker in recombinant fusion proteins. Biotechniques. 2010;49(1): 513-518.

21. Armishaw CJ, Daly NL, Nevin ST, Adams DJ, Craik DJ, Alewood PF. Alpha-selenoconotoxins, a new class of potent alpha7 neuronal nicotinic receptor antagonists. J Biol Chem. 2006;281(20):14136-14143.

22. Hondal RJ, Nilsson BL, Raines RT. Selenocyteine in native chemical ligation and expressed protein ligation. J Am Chem Soc. 2001;123(21): 5140-5141.

23. Muttenthaler M, Nevin ST, Grishin AA, et al. Solving the $\alpha$-conotoxin folding problem: efficient selenium-directed on-resin generation of more potent and stable nicotinic acetylcholine receptor antagonists. J Am Chem Soc. 2010;132(10):3514-3522.

24. Pegoraro S, Fiori S, Rudolph-Böhner S, Watanabe TX, Moroder L. Isomorphous replacement of cystine with selenocystine in endothelin: oxidative refolding, biological and conformational properties of [Sec3,Sec11,Nle7]-endothelin-1. J Mol Biol. 1998;284(3):779-792.

25. Zeng H. Selenium as an essential micronutrient: roles in cell cycle and apoptosis. Molecules. 2009;14(3):1263-1278.

26. Laakkonen P, Porkka K, Hoffman JA, Ruoslahti E. A tumor-homing peptide with a targeting specificity related to lymphatic vessels. Nat Med. 2002;8(7):751-755.

27. Fogal V, Zhang LL, Krajewski S, Ruoslahti E. Mitochondrial/cell surface protein $\mathrm{p} 32 / \mathrm{gC} 1 \mathrm{qR}$ as molecular target in tumor cells and tumor stroma. Cancer Res. 2008;68(17):7210-7218.

28. Yan Z, Zhan C, Wen Z, et al. LyP-1-conjugated doxorubicin-loaded liposomes suppress lymphatic metastasis by inhibiting lymph node metastases and destroying tumor lymphatics. Nanotechnology. 2011; 22(41):415103.

29. Yan Z, Wang F, Wen Z, et al. LyP-1-conjugated PEGylated liposomes: a carrier system for targeted therapy of lymphatic metastatic tumor. J Control Release. 2012;157(1):118-125.
30. Luo G, Yu X, Jin C, et al. LyP-1-conjugated nanoparticles for targeting drug delivery to lymphatic metastatic tumors. Int Pharm. 2010; 385(1-2):150-156.

31. Uchida M, Kosuge H, Terashima M, et al. Protein cage nanoparticles bearing the LyP-1 peptide for enhanced imaging of macrophage-rich vascular lesions. ACS Nano. 2011;5(4):2493-2502.

32. Wang ZH, Yu Y, Ma J, et al. LyP-1 modification to enhance delivery of artemisinin or fluorescent probe loaded polymeric micelles to highly metastatic tumor and its lymphatics. Mol Pharm. 2012;9(9): 2646-2657.

33. Guo X, Shi J, Tang Z, Cui D, Zhang Y. Synthesis and biological activity of seleno sunflower trypsin inhibitor analog. Chem Biol Drug Des. 2006;68(6):341-344

34. Cai WB, Chen XY. Preparation of peptide-conjugated quantum dots for tumor vasculature-targeted imaging. Nat Protoc. 2008;3(1): 89-96.

35. Nallamothu R, Wood GC, Pattillo CB, et al. A tumor vasculature targeted liposome delivery system for combretastatin A4: design, characterization, and in vitro evalution. AAPS Pharm Sci Tech. 2006;7(2):E32.

36. Durek T, Alewood PF. Preformed selenoesters enable rapid native chemical ligation at intractable sites. Angew Chem Int Ed Engl. 2011; 50(50):12042-12045.

37. Sun Y, Li T, Chen H, et al. Selenium-containing 15-mer peptides with high glutathione peroxidase-like activity. J Biol Chem. 2004;279(36): 37235-37240.

38. Han TS, Zhang MM, Gowd KH, et al. Disulfide-depleted selenoconopeptides: simplified oxidative folding of cysteine-rich peptides. ACS Med Chem Lett. 2010;1(4):140-144.

39. Peqoraro S, Fiori S, Cramer J, Rudolph-Böhner S, Moroder L. The disulfide-coupled folding pathway of apamin as derived from diselenide-quenched analogs and intermediates. Protein Sci. 1999;8(8): 1605-1613.

40. Fiori S, Peqeoraro S, Rudolph-Böhner S, Cramer J, Moroder L. Synthesis and conformational analysis of apamin analogues with natural and non-natural cystine/selenocystine connectivties. Biopolymers. 2000;53(7):550-564.

41. Raffa RB. Diselenium, instead of disulfide, bonded analogs of conotoxins: novel synthesis and pharmacotherapeutic potential. Life Sci. 2010;87(15-16):451-456.

42. Understanding the mechanical biology of life's bonds [webpage on the Internet]. New York: Columbia University; 2011. Available from: http://news.columbia.edu/fernandez. Accessed December 21, 2011.

43. Lee SM, Lee EJ, Hong HY, et al. Targeting bladder tumor cells in vivo and in the urine with a peptide identified by phage display. Mol Cancer Res. 2007;5(1):11-19.

44. Witt H, Hajdin K, Iljin K, et al. Identification of a rhabdomyosarcoma targeting peptide by phage display with sequence similarities to the tumor lymphatic-homing peptide LyP-1. Int J Cancer. 2009;124(9): 2026-2032.

45. Dong J, Liu W, Jiang A, Zhang KJ, Chen MQ. A novel peptide, selected from phage display library of random peptides, can efficiently target into human breast cancer cell. Chin Sci Bull. 2008;53(6):860-867.

46. Bickel U, Kang YS, Pardridge WM. In vivo cleavability of a disulfide-based chimeric opioid peptide in rat brain. Bioconjug Chem. 1995;6(2): 211-218.

47. Pirollo KF, Chang EH. Does a targeting ligand influence nanoparticle tumor localization or uptake? Trends Biotechnol. 2008;26(10): $552-558$. 


\section{Supplementary materials}

Validation study of high performance liquid chromatography (HPLC) method

Since the amount of active agent (doxorubicin) was evaluated by HPLC, a validation study is required. According to the method of China Pharmacopoeia (2010 edition), the mobile phase consisted of a mixture of acetonitrile-methanol-water (containing $1.44 \mathrm{~g}$ sodium dodecyl sulfate and $0.68 \mathrm{~mL}$ phosphoric acid in $500 \mathrm{~mL}$ water), 500/60/500 (v/v/v). Samples were detected at $254 \mathrm{~nm}$ with a flow rate of $1 \mathrm{~mL} /$ minute. The column temperature was maintained at $40^{\circ} \mathrm{C}$. Daunorubicin was used as the internal standard.

For quantification of doxorubicin, the calibration curve was obtained by plotting the peak-area ratio of doxorubicin to daunorubicin $(\mathrm{y})$ versus nominal concentration of doxorubicin (x), as follows: $\mathrm{y}=0.0623 \mathrm{x}+0.0235(\mathrm{r}=0.9997$, $\mathrm{n}=5$ ). The linear range was from 0.1 to $100 \mu \mathrm{g} / \mathrm{mL}$. The accuracy and precision determinations were performed in five replicates at three different concentration levels: low, medium, and high quality control samples. Accuracy of the determination of doxorubicin at the low quality control level was $96.77 \% \pm 2.13 \%$; at the medium quality control the level was $98.11 \% \pm 1.64 \%$; and at the high quality control it was $97.18 \% \pm 1.01 \%$. Within-day and between-day precisions were all below $4.0 \%$.

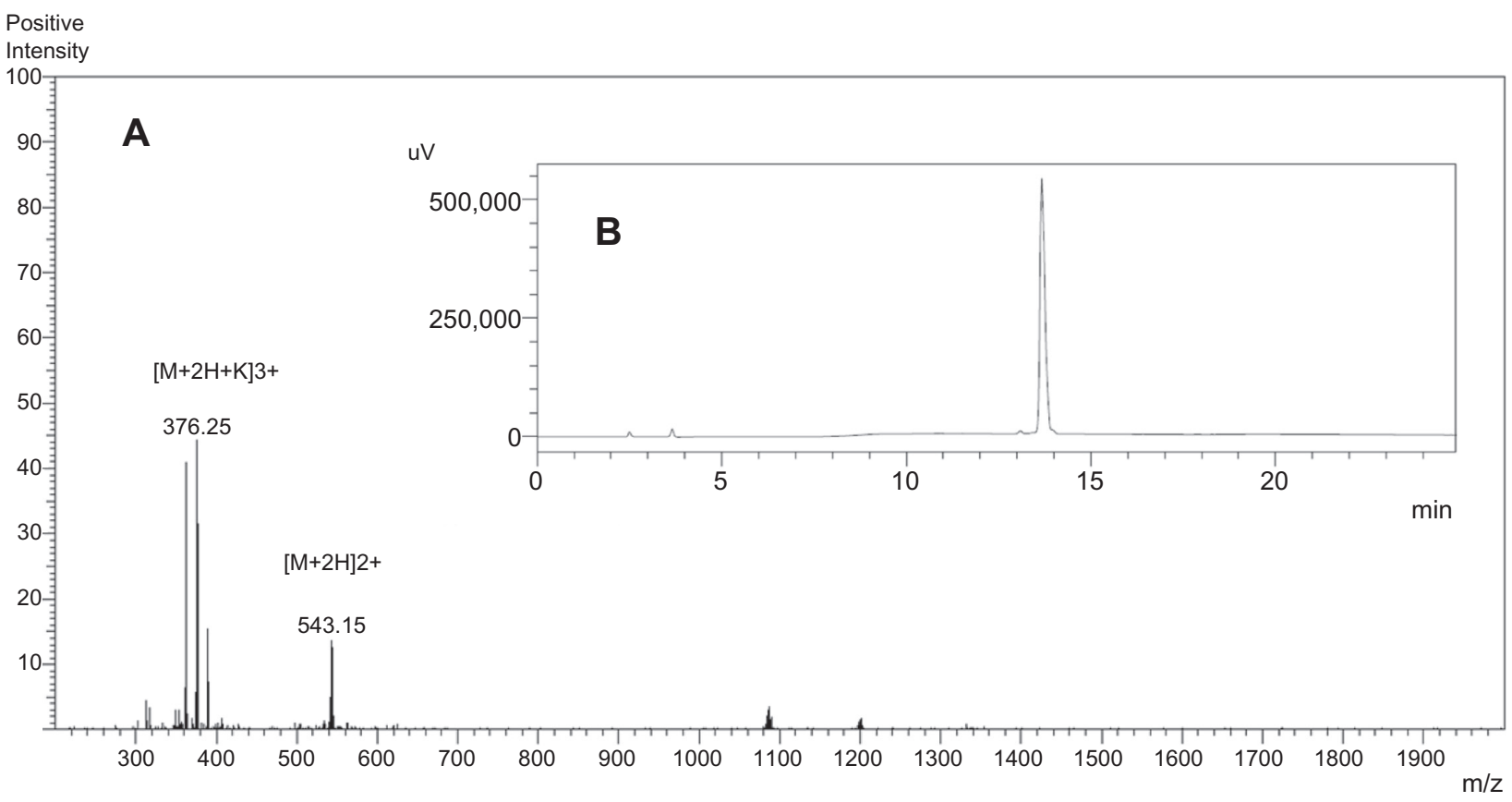

Figure SI Syp-I peptide analyzed by (A) HPLC and (B) ESI-MS.

Notes: HPLC analysis was performed at $40^{\circ} \mathrm{C}$ on a C4 column using a linear gradient of $5 \%-65 \%$ acetonitrile containing $0.1 \%$ TFA at a flow rate of I mL/minute for 30 minutes.

Abbreviations: HPLC, high-performance liquid chromatography; ESI-MS, electrospray ionization mass spectrometry; TFA, trifluoroacetic acid. 
Table SI Kinetic parameters and affinities of interaction between peptide and p32

\begin{tabular}{llll}
\hline Peptide & Ka (I/Ms) & Kd (I/second) & Kd (M) \\
\hline Syp-I & $3.09 E 4$ & $5.73 \mathrm{E}-4$ & $18.54 \mathrm{E}-8$ \\
Lyp-I & $2.02 \mathrm{E} 4$ & $2.14 \mathrm{E}-4$ & $10.59 \mathrm{E}-8$ \\
\hline
\end{tabular}

International Journal of Nanomedicine

\section{Publish your work in this journal}

The International Journal of Nanomedicine is an international, peerreviewed journal focusing on the application of nanotechnology in diagnostics, therapeutics, and drug delivery systems throughout the biomedical field. This journal is indexed on PubMed Central, MedLine, CAS, SciSearch ${ }^{\circledR}$, Current Contents ${ }^{\circledR} /$ Clinical Medicine,
Table S2 The properties of FAM, DiR, or DOX-loaded liposomes with or without peptide modification

\begin{tabular}{lllll}
\hline Formulation & $\begin{array}{l}\text { Vesicle } \\
\text { size } \\
(\mathbf{n m})\end{array}$ & $\begin{array}{l}\text { Polydispersity } \\
\text { index }\end{array}$ & $\begin{array}{l}\text { Zeta } \\
\text { potential } \\
(\mathbf{m v})\end{array}$ & $\begin{array}{l}\text { Encapsulation } \\
\text { efficiency }(\%)\end{array}$ \\
\hline P-LS/DOX & 83.28 & 0.095 & -26.2 & $95.8 \%$ \\
L-P-LS/DOX & 89.56 & 0.17 & -22.8 & $97.2 \%$ \\
S-P-LS/DOX & 87.14 & 0.095 & -22.3 & $96.3 \%$ \\
P-LS/FAM & 98.04 & 0.302 & -14.2 & - \\
L-P-LS/FAM & 95.50 & 0.163 & -12.8 & - \\
S-P-LS/FAM & 97.25 & 0.207 & -12.6 & - \\
P-LS/DiR & 80.84 & 0.186 & -18.6 & - \\
L-P-LS/DiR & 82.66 & 0.202 & -16.4 & - \\
S-P-LS/DiR & 85.36 & 0.179 & -18.2 & - \\
\hline
\end{tabular}

Note: Data are represented as mean values $(n=3)$.

Abbreviations: FAM, 5-carboxyfluorescein; DiR, I,I'-dioctadecyl-3,3,3,3' tetramethyl indotricarbocyanine iodide; DOX, doxorubicin; P-LS/DOX, PEGliposomes loading DOX; L-P-LS/DOX, LyP-I-PEG-liposomes loading DOX; S-PLS/DOX, Syp-I-PEG-liposomes loading DOX; P-LS/FAM, PEG-liposomes loading FAM; L-P-LS/FAM, Lyp-I-PEG-liposomes loading FAM; S-P-LS/FAM, Syp-I-PEGliposomes loading FAM; P-LS/DiR, PEG-liposomes loading DiR; L-P-LS/DiR, Lyp-IPEG-liposomes loading DiR; S-P-LS/DiR, Syp-I-PEG-liposomes loading DiR; PEG, polyethylene glycol; SD, standard deviation.
Journal Citation Reports/Science Edition, EMBase, Scopus and the Elsevier Bibliographic databases. The manuscript management system is completely online and includes a very quick and fair peer-review system, which is all easy to use. Visit http://www.dovepress.com/ testimonials.php to read real quotes from published authors. 